\title{
Splitting Schedules for Internet Broadcast Communication
}

\author{
Kevin Foltz, Student Member, IEEE, and Jehoshua Bruck, Fellow, IEEE
}

\begin{abstract}
The broadcast disk provides an effective way to transmit information from a server to many clients. Work has been done to schedule the broadcast of information in a way that minimizes the expected waiting time of the clients. Much of this work has treated the information as indivisible blocks. We look at splitting items into smaller pieces that need not be broadcast consecutively. This allows us to have better schedules with lower expected waiting times. We look at the case of two items of the same length, each split into two halves, and show how to achieve optimal performance. We prove the surprising result that there are only two possible types of optimal cyclic schedules for items 1 and 2 . These start with 1122 and 122122 . For example, with demand probabilities $p_{1}=0.08$ and $p_{2}=0.92$, the best order to use in broadcasting the halves of items 1 and 2 is a cyclic schedule with cycle 122122222 . We also look at items of different lengths and show that much of the analysis remains the same, resulting in a similar set of optimal schedules.
\end{abstract}

Index Terms-Asymmetric communication, broadcast disk, mobile computing, scheduling, splitting, wireless.

\section{INTRODUCTION}

A S MOBILE computing gains popularity, it becomes increas- ingly important to find efficient methods of communicating with mobile clients. In general, the mobile clients have considerably less outgoing bandwidth than incoming bandwidth, making communication highly asymmetric. Web browsing is a good example of this situation. A person browsing the Web typically receives a lot of information and sends very little. We will describe an efficient way to send information to portable Web browsers.

The broadcast disk is a way to send information to many clients at the same time. Using this scheme, data is broadcast through the air in a cyclic fashion. When a client wants some data, it listens to this broadcast stream until it receives the desired data. If the desired data is not in the broadcast cycle, other means are used to retrieve the data. Essentially, the broadcast disk acts as a common cache for many clients, where data in this cache is made available cyclically, according to the broadcast schedule. Our goal is to schedule the broadcast of information in a way that minimizes the expected waiting time of the clients.

Vaidya and Hameed [5], [6], [9] derived the optimal broadcast frequencies of items within a schedule as a function of their

Manuscript received July 25, 2000; revised September 18, 2001

The authors are with PARADISE Laboratory, California Institute of Technology, Pasadena, CA 91125 USA (e-mail: kfoltz@ paradise.caltech.edu; bruck @ paradise.caltech.edu).

Communicated by T. E. Fuja, Associate Editor At-Large.

Publisher Item Identifier S 0018-9448(02)00301-2. demand probabilities $p_{i}$ and lengths $l_{i}$. They showed that to minimize expected waiting time, the frequencies of broadcast $f_{i}$ should be proportional to $\sqrt{\frac{p_{i}}{l_{i}}}$. This led to an algorithm that attempted to achieve these relative frequencies. This algorithm is good because it is computationally simple and works for an arbitrary number of broadcast items with arbitrary lengths and demand probabilities.

Jiang and Vaidya [7] discuss ways to minimize the variance of the response time. They also propose a way to trade off between minimizing the mean and minimizing the variance of the response time. Aksoy and Franklin [1] discuss scheduling the broadcast of information based on client requests. They consider such metrics as average and worst case performance, scheduling overhead, and robustness in the presence of environmental changes. Bestavros [3] describes a way to add fault tolerance to broadcast disks by sending parity information in addition to data. Bar-Noy, Bhatia, Naor, and Schieber [2] look at scheduling in general, and show that there is an optimal cyclic schedule for a broadcast disk, and finding it is NP-hard. Leong and Si [8] discuss how to choose which items to broadcast, using ideas of cache management. Franklin, Zdonik, Acharya, and Alonso [4], [11] also discuss aspects of broadcast disks. Xu [10] looks at splitting items in the context of on-demand streaming media and shows how to dramatically reduce waiting times by splitting the data and sending it out of sequence.

We examine the scheduling of items for a broadcast disk. We represent a broadcast schedule for two items by a sequence of 1's and 2's, where "1" represents the broadcast of the first item, and "2" represents the broadcast of the second item. In this paper, we think of each item as consisting of two halves, and a 1 or 2 will represent one of these halves, not the entire item. For example, a schedule in which the two items are broadcast in their entirety alternately is $112211221122 \ldots$ and not $121212 \ldots$. since we need two halves of each item to broadcast the entire item.

Broadcast schedules are cyclic, so we will represent them by one of their cycles. Since each item has two halves, we assume these halves are broadcast alternately. The schedule 122 , for example, should really be written as $1_{A} 2_{A} 2_{B} 1_{B} 2_{A} 2_{B}$, where $1_{A}$ and $1_{B}$ are the two halves of item 1 , and $2_{A}$ and $2_{B}$ are the two halves of item 2 . This would more accurately represent one period, but we shorten the representation to 122 with the understanding that the two halves of each item are broadcast alternately. As a shorthand representation, we will sometimes use exponents to indicate repeated items. For example, $12^{2}$ represents 122 and $1^{2} 2^{3} 12$ represents 1122212 .

Another representation of a schedule that we will use is based on the number of 2's between consecutive 1's in 
the schedule. We use a bracketed sequence of numbers that represent the number of 2's between each consecutive pair of 1 's. For example, $[0,2]$ represents the schedule 1122 , and $[0,0,1,3,2]$ represents 11121222122 . We use the notation $S^{C}$ to represent the complement of $S$, the schedule $S$ with 1's and 2's swapped. For example, if $S=12211112$, then $S^{C}=21122221$. We use $S^{R}$ to represent the reversal of $S$. With $S$ as above, $S^{R}=21111221$.

Sometimes we want to indicate that a certain instance of an item may or may not be present in a schedule. Parentheses will be used to indicate the possible presence of an instance of an item in a schedule. For example, a schedule in which we know only that item 2 is never broadcast twice consecutively could be represented as $1(2) 1(2) \ldots 1(2)$.

We define a useful function dealing with expected waiting times.

Definition 1: $\operatorname{EWT}\left(S, p_{1}\right)$ is the expected waiting time using schedule $S$ with demand probabilities $p_{1}$ and $p_{2}=1-p_{1}$, assuming two items, each split into two halves.

We assume that the broadcast data is static, so the data in each item is identical from one transmission to the next. We also assume that an item or piece of an item (when we split) must be read starting at the beginning. We compute waiting time for an item by looking at how long a client must wait to get all of the desired item, starting at a particular point within the broadcast cycle. We average over all starting points to get the EWT for that item. We do this for each item and weight these times by the demand probabilities to get the overall EWT.

This is our metric for evaluating schedules. The lower the value of EWT $\left(S, p_{1}\right)$, the better schedule $S$ is for demand probabilities $p_{1}$ and $p_{2}=1-p_{1}$.

Vaidya and Hameed assume a uniform spacing of items within a schedule (which is the optimal spacing, when achievable) to derive their broadcast frequencies. However, in most cases it is not possible to achieve these frequencies with uniform spacing, since the individual schedules for the different items usually do not mesh together perfectly. This can lead to a large deviation of the EWT from the optimal time.

As an example, consider two items, one that is accessed frequently and another that is accessed less frequently. Suppose $l_{1}=1, p_{1}=0.2, l_{2}=1, p_{2}=0.8$. Then, according to Vaidya and Hameed, we want $f_{1}=\frac{1}{3}, f_{2}=\frac{2}{3}$. We want to broadcast with equal spacing, so we should schedule items 1 and 2 as in Fig. 1(a) and (b). In Fig. 1(a), item 1 has a waiting time ranging from 0 to 3 , with an average of $\frac{3}{2}$. In Fig. 1(b), item 2 has a waiting time varying from 0 to $\frac{3}{2}$, with an average of $\frac{3}{4}$. If we could somehow merge these two schedules together, we would achieve an EWT of $0.2 \cdot \frac{3}{2}+0.8 \cdot \frac{3}{4}=\frac{9}{10}$. However, it is impossible to merge these schedules together while preserving the property of uniform spacing. The scheduling algorithm described by Vaidya and Hameed gives us one of the schedules in Fig. 1(c) or (d), depending on how we break ties. These have expected waiting times 1 and $\frac{29}{30}$.

To address the problem of merging schedules, we relax the restriction used by Vaidya and Hameed of broadcasting an item in one piece, and look at splitting items into subitems and scheduling the broadcast of these subitems. We look at the (a)

\begin{tabular}{|l|l|l|l|l|l|l|l|}
\hline 1 & & 1 & & 1 & & 1 & \\
\hline
\end{tabular}

(b)

\begin{tabular}{|l|l|l|l|l|l|l|l|l|l|l|l|l|l|l|l|}
\hline 2 & & 2 & & 2 & & 2 & & 2 & & 2 & & 2 & & 2 & \\
\hline
\end{tabular}

(c)

\begin{tabular}{|l|l|l|l|l|l|l|l|l|l|l|l|}
\hline 1 & 2 & 2 & 1 & 2 & 2 & 1 & 2 & 2 & 1 & 2 & 2 \\
\hline
\end{tabular}

(d)

\begin{tabular}{|l|l|l|l|l|l|l|l|l|l|l|l|}
\hline 1 & 2 & 1 & 2 & 1 & 2 & 1 & 2 & 1 & 2 & 1 & 2 \\
\hline
\end{tabular}

(e)

\begin{tabular}{|l|l|l|l|l|l|l|l|l|l|l|l|l|l|l|l|l|l|l|l|l|l|l|l|}
\hline 1 & 2 & 2 & 1 & 2 & 2 & 1 & 2 & 2 & 1 & 2 & 2 & 1 & 2 & 2 & 1 & 2 & 2 & 1 & 2 & 2 & 1 & 2 & 2 \\
\hline
\end{tabular}

Fig. 1. (a) Ideal scheduling for item 1, (b) ideal scheduling for item 2, (c) and (d) actual schedules generated using Vaidya and Hameed's algorithm, (e) our schedule using splitting.

specific case of two items of the same length, each split into two subitems. We find that the optimal schedule for the example above is the schedule in Fig. 1(e), with expected waiting time $\frac{49}{60}$. Note that this result, when splitting is allowed, is better than even the unachievable theoretical limit of $\frac{9}{10}$ when splitting is not allowed.

In general, we show the following.

Theorem 1: For two items of the same length, each split into two halves, the broadcast schedule that minimizes expected waiting time is

$$
\begin{array}{r}
1122 \overbrace{2 \ldots 2}^{n}, n=\operatorname{Max}\left(0,\left[\frac{-9+\sqrt{-47+\frac{40}{p_{1}}}}{2}\right]\right), \\
\text { if } p_{1} \in\left[\frac{1}{5}, \frac{1}{2}\right] \\
122122 \overbrace{2 \ldots 2}^{n}, n=\operatorname{Max}\left(0,\left[\frac{-13+\sqrt{-103+\frac{32}{p_{1}}}}{2}\right]\right), \\
\text { if } p_{1} \in\left(0, \frac{1}{5}\right) .
\end{array}
$$

In Section II, we present lemmas useful in proving Theorem 1. We discuss the implications of each lemma, leaving their proofs for the Appendix. In Section III, we prove the theorem. We describe how to reduce the search for optimal schedules from all schedules to a smaller set of irreducible schedules. We determine which schedules are in this set. Then, we compare these with each other and see that the schedules listed in Theorem 1 are optimal on their respective intervals. We also show the optimal schedules without splitting, for comparison.

In Section IV, we consider items of different lengths. We present numerical results that suggest that the optimal schedules for different length items are the same as those for items of the same length. In Section V, we discuss future directions for 
research. The Appendix contains proofs of the lemmas stated in Section II.

\section{THE LEMMAS}

These lemmas provide ways of manipulating schedules into similar but better schedules. Their proofs are in the Appendix. In Lemmas 1 and 2, we assume two items, each split into two halves, with $l_{1}=1$.

Lemma 1 (The Rearrangement Lemma): The following hold for all values of $p_{1}$ :

a)

$$
\begin{aligned}
\operatorname{EWT}\left(S^{\prime}, p_{1}\right) \leq & \operatorname{EWT}\left(S, p_{1}\right) \text { where } \\
S= & {[a, b, c, \ldots, d, e, f, \ldots] } \\
S^{\prime}= & {[a, b-1, c, \ldots, d, e+1, f, \ldots] \text { and } } \\
& (a+b+c)-(d+e+f) \geq 1 .
\end{aligned}
$$

b)

$\operatorname{EWT}\left(T^{\prime}, p_{1}\right) \leq \operatorname{EWT}\left(T, p_{1}\right)$ where

$$
\begin{aligned}
& T=\ldots 1 \overbrace{22 \ldots 222}^{n \geq 2} 121 \overbrace{2}^{x=0,1} 1 \overbrace{2}^{y=0,1} 1 \ldots \\
& T^{\prime}=\ldots 1 \overbrace{22 \ldots 222}^{n} 112 \overbrace{2}^{x} 1 \overbrace{2}^{y} 1 \ldots .
\end{aligned}
$$

c)

$\operatorname{EWT}\left(U^{\prime}, p_{1}\right) \leq \operatorname{EWT}\left(U, p_{1}\right)$ where

$$
\begin{aligned}
U & =\ldots \overbrace{1 \ldots 1}^{r \geq 1} \overbrace{2 \ldots 2}^{s \geq 1} \overbrace{11 \ldots 11}^{t \geq 3} \overbrace{22 \ldots 22}^{u \geq 3} 1 \ldots \\
U^{\prime} & =\ldots \overbrace{1 \ldots 1}^{r} \overbrace{2 \ldots 2}^{s} \overbrace{11 \ldots 1}^{t-1} 21 \overbrace{2 \ldots 22}^{u-1} 1 \ldots .
\end{aligned}
$$

d)

$\operatorname{EWT}\left(V^{\prime}, p_{1}\right)=\operatorname{EWT}\left(V, p_{1}\right)$ where

$V=\overbrace{1 \ldots 1}^{a_{1}} \overbrace{2 \ldots 2}^{b_{1}} \overbrace{1 \ldots 1}^{a_{2}} \overbrace{2 \ldots 2}^{b_{2}} \ldots \overbrace{1 \ldots 1}^{a_{k-1}} \overbrace{2 \ldots 2}^{b_{k-1}} \overbrace{1 \ldots 1}^{a_{k}} \overbrace{2 \ldots 2}^{b_{k}}$

$V^{\prime}=\overbrace{2 \ldots 2}^{b_{k}} \overbrace{1 \ldots 1}^{a_{k}} \overbrace{2 \ldots 2}^{b_{k-1}} \overbrace{1 \ldots 1}^{a_{k-1}} \ldots \overbrace{2 \ldots 2}^{b_{2}} \overbrace{1 \ldots 1}^{a_{2}} \overbrace{2 \ldots 2}^{b_{1}} \overbrace{1 \ldots 1}^{a_{1}}$.

Lemma 2 (Corollary to Lemma 1 Part a)): If, instead of

$$
S=[a, b, c, \ldots, d, e, f, \ldots]
$$

$S$ is one of

$$
\begin{aligned}
& {[a, b, c=d, e, f, \ldots]} \\
& {[a, b=d, c=e, f, \ldots]} \\
& {[a=f, b, c=d, e]}
\end{aligned}
$$

or

$$
[a=e, b=f, c=d]
$$

then Lemma 1 part a) still holds, where $S^{\prime}$ is $S$ with $b$ decremented by 1 and $e$ incremented by 1 .

Lemma 1 part a) and its corollary tell us that it is generally not good to have strings of 2 's of significantly different lengths.
More specifically, for each string of two or more 2's, we add its length to the sum of the lengths of the adjacent strings of 2's. These sums should be as close to each other as possible (equal or within 1) for all strings of two or more 2's in the schedule.

For example, for the schedule

\section{1}

consider the two long strings of 2's of lengths 5 and 7 . Their adjacent strings of 2's have lengths 1 and 0 and 2 and 1 , respectively, giving sums of $5+1+0=6$ and $7+2+1=10$. Since $10-6=4 \geq 1$, we can move a 2 from the string of length 7 to the string of length 5 , giving the new and better schedule

$$
1212222221121221222222121
$$

with sums $6+1+0=7$ and $6+2+1=9$. Since our new sums differ by 2 , we can move another 2 to get an even better schedule

$$
1212222222112122122222121
$$

with sums 8 and 8 . However, moving another 2 will not give us a better schedule, since $8-8=0 \nsupseteq 1$.

The corollary allows us to eliminate adjacent long strings of 2 's. We do this by performing the operation in the lemma. We call our adjacent strings the $b$ - and $e$-length strings. After multiple applications of the lemma (move a 2 from one of these strings to the other), we get one of the strings down to length 2 .

As an example, consider the schedule

$$
1212222212222112 .
$$

We have adjacent strings of 2's of lengths 5 and 4 . By considering these strings as we did above, we get sums of $5+1+4=10$ and $4+5+0=9$. So, if we move a 2 from the string of length 5 to the string of length 4 , we improve our schedule. However, unlike before, our sums remain the same at $4+1+5=10$ and $5+4+0=9$, so we can repeat this procedure until we are left with only two 2 's in the first string and a new schedule of

$$
1212212222222112 .
$$

Lemma1 part b) applies when we have blocks of 1's, possibly with some single 2's in them, bounded on both sides by at least two 2's. This lemma tells us that if the beginning or end of the block is 121 , it is better to shift the 2 toward the inside of the block to get 112 [rest of block] or [rest of block] 211 , when there is a total of at least four 1's in the block.

An example of this is the schedule

$$
12212111212112222 .
$$

This contains the string 1211121211 , which is a block of seven 1 's with only single 2 's, bordered on both sides by 22 . Lemma 1 part b) tells us that it is better to have 112 than 121 at the beginning of this string. It is better to rearrange the start of the string to get 1121121211, for a new and better schedule of

$$
12211211212112222 .
$$

Lemma 1 part c) tells us that large strings of 1's and 2's should not border each other. It is better to swap the innermost 1 and 2 if we have at least three of each. A simple example of this 
is the schedule 121111122212 . We would be better off using 121111212212 .

Lemma 1 part d) tells us that reversing a schedule does not affect its expected waiting time. So, the schedules 121112222 and 222211121 , for example, have the same expected waiting times.

Lemma 3 (The Splitting Headache Lemma): Split item 1 into $m$ equal pieces of length $\frac{l_{1}}{m}$ and split item 2 into $n$ equal pieces of length $\frac{l_{2}}{n}$.

Suppose schedule $S$ is written as $s_{1} s_{2} \ldots s_{l}$, where each $s_{i}$ is either a 1 or a 2 and represents one piece of item 1 or 2 , respectively. Suppose also that there is a schedule $C=c_{1} c_{2} \cdots c_{k}$, such that $C$ has at least $m 1$ 's and at least $n 2$ 's, and

$$
c_{i}=s_{g_{1}+i \bmod l}=s_{g_{2}+i \bmod l}, \quad \forall i, 1 \leq i \leq k
$$

for some $g_{1} \neq g_{2} \bmod l$. Then

$$
\operatorname{EWT}\left(S, p_{1}\right)=\frac{l_{S_{1}}}{l_{S}} \cdot \operatorname{EWT}\left(S_{1}, p_{1}\right)+\frac{l_{S_{2}}}{l_{S}} \cdot \operatorname{EWT}\left(S_{2}, p_{1}\right)
$$

where

$$
\begin{aligned}
& S_{1}=s_{\left(g_{1}+1\right) \bmod l} s_{\left(g_{1}+2\right) \bmod l} \cdots s_{\left(g_{1}+l_{1}\right) \bmod l} \\
& S_{2}=s_{\left(g_{2}+1\right) \bmod l} s_{\left(g_{2}+2\right) \bmod l} \cdots s_{\left(g_{2}+l_{2}\right) \bmod l}
\end{aligned}
$$

$l_{S_{1}}$ is the length of schedule $S_{1}, l_{S_{2}}$ is the length of schedule $S_{2}$, and $l_{S}$ is the length of schedule $S=l_{S_{1}}+l_{S_{2}}$.

This lemma tells us that under certain conditions we can split a schedule $S$ into two schedules, $S_{1}$ and $S_{2}$. Schedule $S$ will have an expected waiting time that is the weighted mean of the expected waiting times of $S_{1}$ and $S_{2}$. At any value of $p_{1}$, one of $S_{1}$ and $S_{2}$ will have a shorter expected waiting time and the other will have a longer expected waiting time. As a result, we should never use $S$, but instead choose the better of $S_{1}$ and $S_{2}$.

As an example, consider the schedule

$$
11212122221122 .
$$

We rewrite it (by shifting, since we send data cyclically) as

$$
12121222211221 .
$$

This is just 12 concatenated with

$$
121222211221 .
$$

Each of these starts with 1212 , which is a string with two 1's and two 2's. It is easy to see this for the second subschedule. For the first one, think of it not as 12 , but as $121212 \ldots$, which is what we broadcast when we use this schedule. We can split our schedule into these two schedules. We can find the point $p$ where the two schedules have the same expected waiting time, and see that 12 is better for $p_{1}>p$ and 121222211221 is better for $p_{1}<$ $p$. So, instead of using the original schedule 11212122221122 , we should use either 12 or 121222211221 , depending on the value of $p_{1}$.

\section{PROOF OF THEOREM 1}

We begin the proof by classifying schedules into one of two sets. The first set is the "reducible" schedules, the set of all schedules for which one of the lemmas applies to give a strictly better schedule. The second set is the "irreducible" schedules, the set of all schedules for which no lemma can be used to give a strictly better schedule. We see that any schedule will be in exactly one of these two sets. We then look at the set of irreducible schedules, since any reducible schedule is worse than some irreducible schedule and hence not optimal. We compare these irreducible schedules and find that a small subset of them (the set of schedules listed in Theorem 1) forms the set of optimal schedules.

\section{A. The Irreducible Schedules}

Each of the lemmas provides a way to change a schedule to get another equally good or better schedule. We can think of the lemmas as describing actions we can perform on schedules to change them. There are two types of actions. For each type, we assume some fixed value of $p_{1}$.

The first type reduces the expected waiting time of a schedule. This type of action gives a schedule that is strictly better than the original. These actions establish a partial ordering $\rho_{2}$ among schedules. When one schedule can be modified by one of these actions to get a second schedule, the second schedule is less than the first according to $\rho_{2}$.

The second type of action changes the structure of the schedule, but keeps the expected waiting time the same. This type of action does not give a measurably better schedule, but instead identifies schedules that are equal with respect to $\rho_{2}$. When one schedule can be modified by one of these actions to get a second schedule, the two schedules are equal.

There are two orderings of schedules. The first, $\rho_{1}$, is by EWT. Any two schedules can be compared using EWT. This is the ordering we use to define the optimal schedule at any value of $p_{1}$. The optimal schedule is simply the one that is "less than or equal to" all other schedules according to $\rho_{1}$.

The second ordering, $\rho_{2}$, is by the actions described above. Not all schedules can be compared by $\rho_{2}$, just the ones that are the initial and resulting schedules from some action. Any two that can be compared with $\rho_{2}$ will have the same ordering as by $\rho_{1}$, so $\rho_{2}$ is really a subordering of $\rho_{1}$. That is, the set of relationships described by $\rho_{2}$ is a subset of the set of relationships described by $\rho_{1}$.

So, any minimal schedule under $\rho_{1}$ will be a minimal schedule under $\rho_{2}$. Our strategy for determining the optimal schedule (the minimal element under $\rho_{1}$ ) will be to determine the minimal elements under $\rho_{2}$ and then compare them under $\rho_{1}$ to find the optimal schedule.

For ease of referencing the lemmas and their associated transformations, we list the following actions that we can perform on schedules.

A1) $S=\boldsymbol{A B} \rightarrow S_{1}^{\prime}=\boldsymbol{A}, S_{2}^{\prime}=\boldsymbol{B}$, where both $A$ and $B$ start with the same subschedule $C$, which contains at least two 1's and two 2's.

A2)

$$
\begin{aligned}
S=\ldots & 22121 \overbrace{(2) 1(2) 1 \ldots(2) 1}^{n} 22 \ldots \\
& \rightarrow S^{\prime}=\ldots 22112 \overbrace{(2) 1(2) 1 \ldots(2) 1}^{n 1 s} 22 \ldots, n \geq 2 .
\end{aligned}
$$

A3) $S \rightarrow S^{\prime}=S^{R}$. 
A4)

$$
\begin{aligned}
& S=[\ldots, a, \boldsymbol{n}, \boldsymbol{m}, b, \ldots] \\
& \quad \rightarrow S^{\prime}=[\ldots, a, \mathbf{2}, \boldsymbol{m}+n-\mathbf{2}, b, \ldots], \\
& \quad n \geq 2, m \geq 2, b \geq a .
\end{aligned}
$$

A5)

$$
S=[\ldots, a, \boldsymbol{b}, c, \ldots, d, \boldsymbol{e}, f, \ldots]
$$$$
\rightarrow S^{\prime}=[\ldots, a, b-1, c, \ldots, d, e+1, f, \ldots], b \geq 3, e \geq 2 \text {. }
$$

A6)

$$
\begin{aligned}
S & =\ldots 1 \overbrace{22 \ldots 22}^{n 2 s} \overbrace{11 \ldots 11}^{m} 1 s \ldots \\
& \rightarrow S^{\prime}=\ldots 1 \overbrace{22 \ldots 2}^{n-1} 12 \overbrace{11 \ldots 1}^{m-1} 2 \ldots, n \geq 3, m \geq 3 .
\end{aligned}
$$

We use the notation " $N$ " to mean "three or more." Different occurrences of $N$ within a schedule can correspond to different numbers greater than or equal to three. For example, $[0, N, 1,2, N]$ can represent the schedule

$$
[0,3,1,2,3],[0,3,1,2,4] \text {, or }[0,10,1,2,12]
$$

but not

$$
[0,3,1,2,1],[0,0,1,2,2] \text {, or }[0,10,1,2,2] \text {. }
$$

We first show a weaker version of Theorem 1 .

Proposition 1: All optimal schedules are equal to a schedule or the complement of a schedule in the following list:

$[0,1],[0,1,1],[0,1,2],[0,1,2, N],[0,1, N]$,

$[0,1, N, 2],[0,1, N, 2, N],[0,2],[0,2,1, N]$,

$[0,2, N],[0,2, N, 1, N],[0, N],[0, N, 1,2, N]$,

$[0, N, 1, N],[0, N, 1, N, 2, N],[0, N, 2, N]$,

[1], [1, 2], [1, 2,N], [1,N], [1,N, 2,N], [2], [2,N].

Note that the optimal schedules $[0,2],[0,3],[0,4],[2]$, and $[2, N]$ are all included in the list. We show later that these are the best schedules in the list. We now derive this list containing all the irreducible schedules.

First we consider all schedules that do not contain both three consecutive 1's and three consecutive 2's. For now, we assume there are no 111's. We will eliminate certain schedules based on the fact that we can find a better schedule according to $\rho_{2}$. Since we can find a better schedule, the original schedule is reducible, and we can exclude it from the list.

We know that no irreducible schedule can contain more than one each of $1122,2211,1212,2121,1221$, or 2112 , since we could use action A1) on such a schedule to get a better schedule. Here, and in general, we ignore schedules that are optimal at exactly one point, such as the schedule before splitting, at the value of $p_{1}$ where the two subschedules have the same waiting time as the original. This is because the other two schedules are not only optimal at that point, but also at neighboring points.

We cannot have a schedule with two 0's in it, since we would have $[\ldots, 0, \ldots, 0, \ldots]$, which gives us two 2112 's, or $[\ldots, 0,0, \ldots]$, which gives us a 111 . We cannot have a schedule with two 2's in it, since we would have $[\ldots, 2, \ldots, 2, \ldots]$, which gives us two 1221 's. If we have a schedule with two 1's in it, we have two instances of 121 . If both are preceded or followed by something other than
0 , we get two instances of 1212 or 2121 . The only way to prevent this is for one to be preceded by 0 and the other to be followed by 0 . Since we can have at most one 0 , we need to have $[\ldots, 1,0,1, \ldots]$, with only 2 's or larger in the rest of the schedule. But with 2's in the schedule, we can use action A2) to get a better schedule. So, the only irreducible schedule with two 1 's is $[0,1,1]$.

We cannot have a schedule with two adjacent $N$ 's, where $N$ represents some number greater than 2 , since we can use action A4) on such a schedule to get a better schedule. So, the irreducible schedules are $[0,1,1]$ and all schedules that have at most one 0,1 , and 2 , and no adjacent $N$ 's. It is straightforward to list these as follows.

$[0,1,1],[N],[0],[0, N],[1],[1, N],[2],[2, N],[0,1]$, $[0,1, N],[0, N, 1],[0, N, 1, N],[0,2],[0,2, N],[0, N, 2]$, $[0, N, 2, N],[1,2],[1,2, N],[1, N, 2],[1, N, 2, N],[0,1,2]$, $[0,1,2, N],[0,1, N, 2],[0, N, 1,2],[0,1, N, 2, N]$, $[0, N, 1,2, N],[0, N, 1, N, 2],[0,2,1],[0,2,1, N]$, $[0,2, N, 1],[0, N, 2,1],[0,2, N, 1, N],[0, N, 2,1, N]$, $[0, N, 2, N, 1],[1,0,2],[1,0,2, N],[1,0, N, 2],[1, N, 0,2]$, $[1,0, N, 2, N],[1, N, 0,2, N],[1, N, 0, N, 2],[1,2,0]$, $[1,2,0, N],[1,2, N, 0],[1, N, 2,0],[1,2, N, 0, N]$, $[1, N, 2,0, N],[1, N, 2, N, 0],[2,0,1],[2,0,1, N]$, $[2,0, N, 1],[2, N, 0,1],[2,0, N, 1, N],[2, N, 0,1, N]$, $[2, N, 0, N, 1],[2,1,0],[2,1,0, N],[2,1, N, 0]$, $[2, N, 1,0],[2,1, N, 0, N],[2, N, 1,0, N],[2, N, 1, N, 0]$, $[0, N, 1, N, 2, N],[0, N, 2, N, 1, N]$.

We can eliminate repetitions of the same schedule using periodicity of the schedules. For example, $[0,2, N, 1]=[1,0,2, N]$. We can use action A3) to eliminate reversals. We also eliminate [0], since it does not contain item 2). When we do this, we get the following list of schedules:

$[0,1],[0,1,1],[0,1,2],[0,1,2, N],[0,1, N],[0,1, N, 2]$,

$[0,1, N, 2, N],[0,2],[0,2,1, N],[0,2, N],[0,2, N, 1, N]$,

$[0, N],[0, N, 1,2, N],[0, N, 1, N],[0, N, 1, N, 2, N]$

$[0, N, 2, N],[1],[1,2],[1,2, N],[1, N],[1, N, 2, N],[2],[2, N]$.

If we allow 111 and not 222 , we get the complements of the schedules in this list. Thus, the set of these schedules and their complements contains all irreducible schedules that do not have both a sequence of 111 and a sequence of 222 .

We now consider schedules with both 111 and 222 sequences. We can decompose any such schedule into a sequence of subschedules that begin and end with either 111 or 222 , and have no sequences of three or more 1's or 2's other than at their beginning or end. We will now derive the set of irreducible subschedules that can be combined to form irreducible schedules.

To do this, we start with the sequence 1112 and extend this schedule one piece at a time until we reach either a $111 \ldots 111$ subschedule, a $111 \ldots 222$ subschedule, or a reducible subschedule (a subschedule such that any schedule containing it is reducible). For each position, we can choose either 1 or 2 , so we search all possibilities using a binary tree. This is diagramed in Fig. 2. 


\section{Tree used to generate schedules with both 111's and 222's}

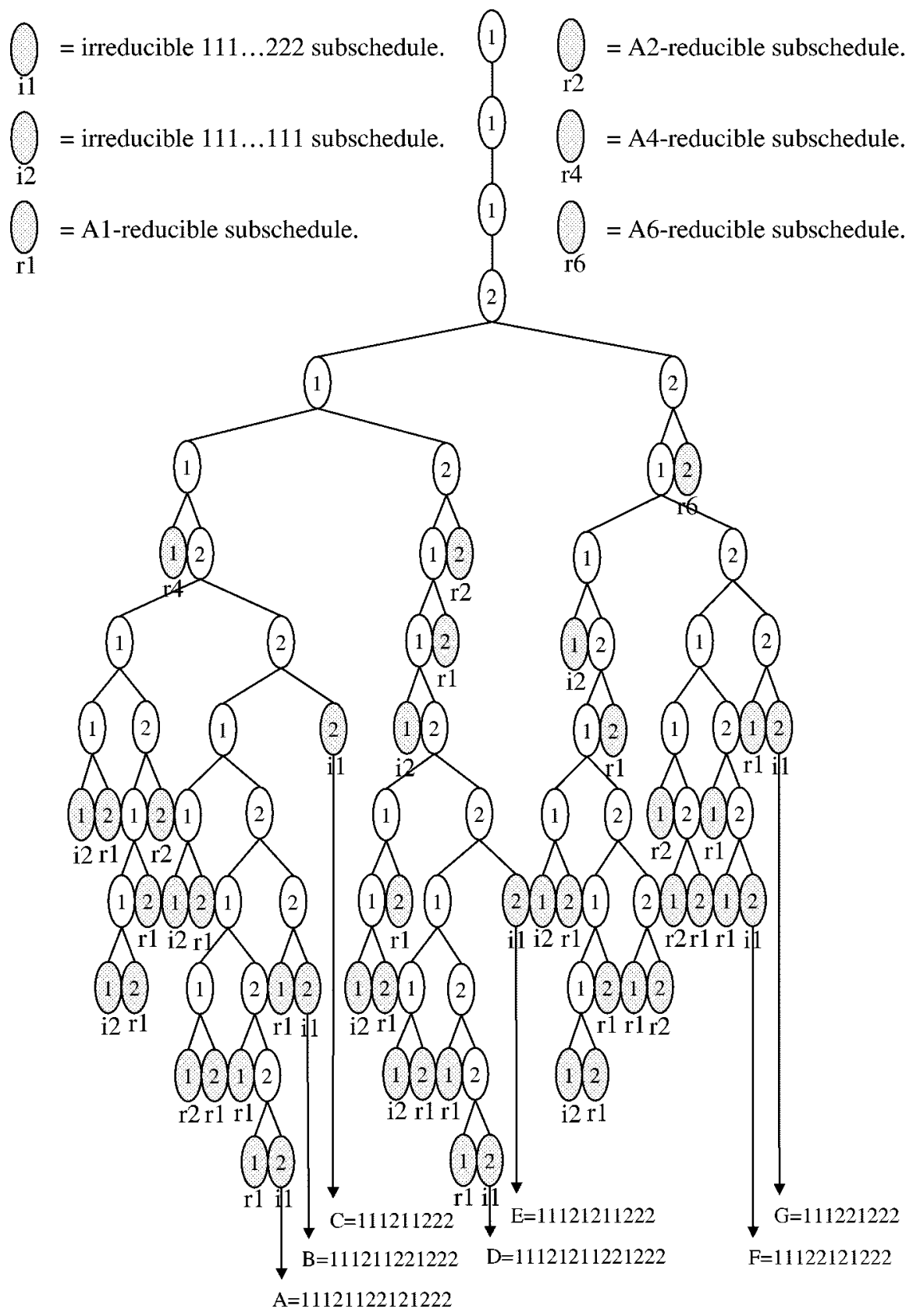

Fig. 2. Tree of schedules.

From this tree, we see that there are seven irreducible $111 \ldots 222$ subschedules. We label them A through G. The irreducible $222 \ldots 111$ subschedules are simply the complements of the irreducible $111 \ldots 222$ subschedules. We will call these $\overline{\mathrm{A}}$ through $\overline{\mathrm{G}}$. We now generate $111 \ldots 222 \ldots$ schedules by combining these $111 \ldots 222$ and $222 \ldots 111$ subschedules. We use action A1) to eliminate reducible schedules and we find that the only irreducible combinations are $\mathrm{C} \overline{\mathrm{C}}, \overline{\mathrm{E}}, \mathrm{E} \overline{\mathrm{C}}, \overline{\mathrm{F}} \overline{\mathrm{G}}$, $\mathrm{G} \overline{\mathrm{F}}$, and $\mathrm{G} \overline{\mathrm{G}}$.

Of these, only $\mathrm{C} \overline{\mathrm{C}}$ and $\mathrm{G} \overline{\mathrm{G}}$ do not contain all six possible patterns of length four that have two each of item 1) and item 2). In these, we can replace 111 with $111 \ldots 111$, if the $111 \ldots 111$ subschedule does not have any of the same patterns in it as the starting schedule. We find that the only such $111 \ldots 111$ subschedule is 111212111 . When this subschedule is added to $\mathrm{C} \overline{\mathrm{C}}$ and $\mathrm{G} \overline{\mathrm{G}}$, each resulting schedule contains all six patterns. So, the only possible schedules with both 111 and 222 are: $\mathrm{C} \overline{\mathrm{C}}$, $\mathrm{C} \overline{\mathrm{E}}, \mathrm{E} \overline{\mathrm{C}}, \mathrm{F} \overline{\mathrm{G}}, \mathrm{G} \overline{\mathrm{F}}, \mathrm{G} \overline{\mathrm{G}}, \mathrm{C}^{\prime} \overline{\mathrm{C}}, \mathrm{G}^{\prime} \overline{\mathrm{G}}, \mathrm{C}_{\mathrm{C}^{\prime}}$, and $\mathrm{G}_{\mathrm{G}}{ }^{\prime}$, where $\mathrm{C}^{\prime}$ is $\mathrm{C}$ with 111 replaced by 111212111 and $\mathrm{G}^{\prime}$ is $\mathrm{G}$ with 111 replaced by 111212111.

We need only consider $C \overline{\mathrm{C}}, \mathrm{C} \overline{\mathrm{E}}$, and $\mathrm{C}^{\prime} \overline{\mathrm{C}}$, since $\mathrm{E} \overline{\mathrm{C}}=(\mathrm{C} \overline{\mathrm{E}})^{C}, \mathrm{~F} \overline{\mathrm{G}}=(\mathrm{C} \overline{\mathrm{E}})^{R}, \mathrm{G} \overline{\mathrm{F}}=(\mathrm{C} \overline{\mathrm{E}})^{C R}, \mathrm{G} \overline{\mathrm{G}}=(\mathrm{C} \overline{\mathrm{C}})^{C R}$
$\mathrm{G}^{\prime} \overline{\mathrm{G}}=\left(\mathrm{C}^{\prime} \overline{\mathrm{C}}\right)^{R}, \mathrm{C}^{\prime}=\left(\mathrm{C}^{\prime} \overline{\mathrm{C}}\right)^{C}$, and $\mathrm{G}^{\prime}=\left(\mathrm{C}^{\prime} \overline{\mathrm{C}}\right)^{C R}$.

We use action A4) on

$$
\overline{\mathrm{C}}=111211222122
$$




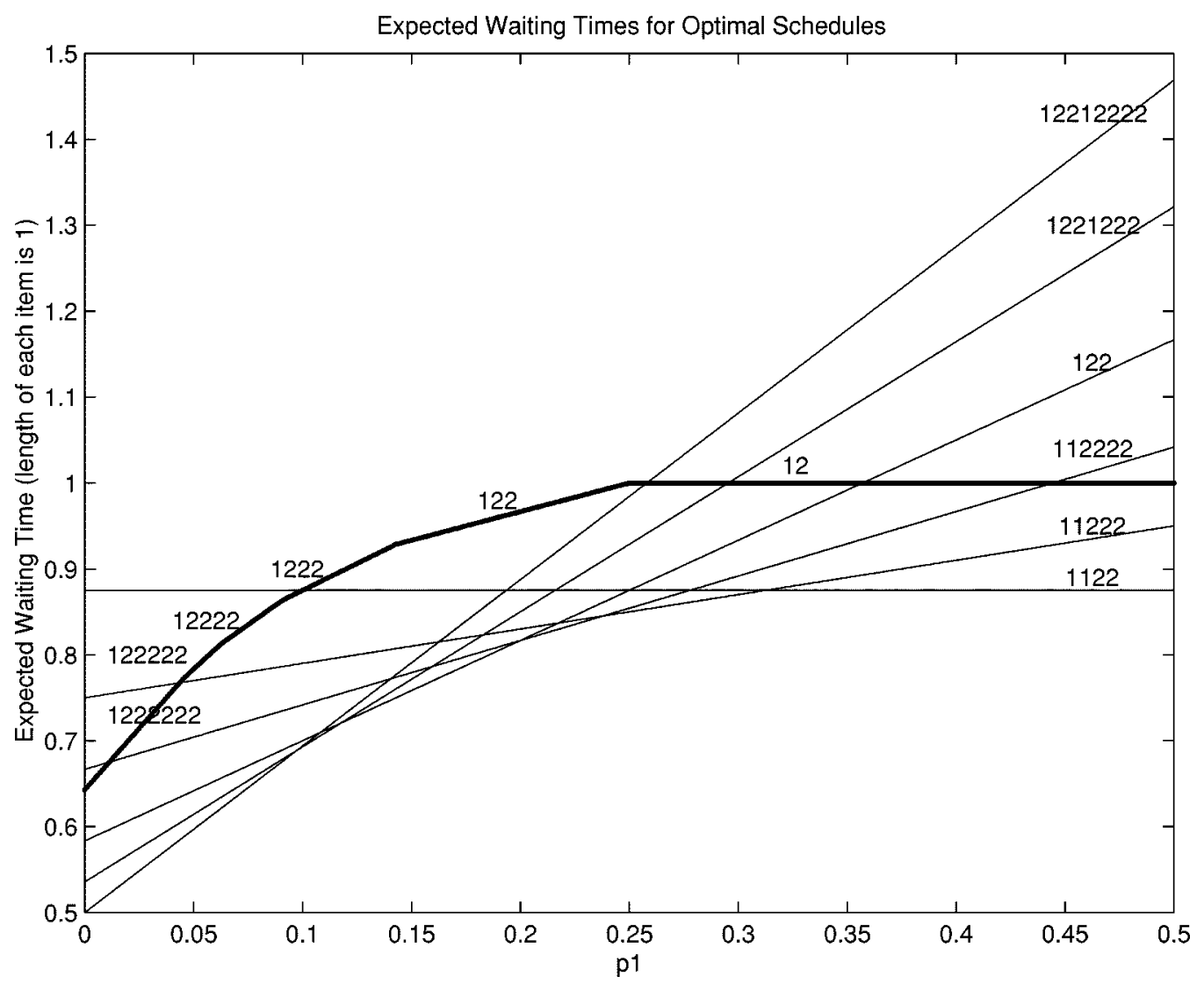

Fig. 3. Expected waiting time versus $p_{1}$ for some of the optimal schedules. The lines are for splitting, the solid piecewise-linear curve is for no splitting.

to get 111211221222 . We then use action A6) to get the schedule 211211221221 , to which we can apply action A2). We can do the same thing to

$$
\mathrm{C} \overline{\mathrm{E}}=11121122212122
$$

and

$$
\mathrm{C}^{\prime} \overline{\mathrm{C}}=111212111211222122
$$

applying action A4) to give

$$
11211122212122 \text { and } 111212112111222122
$$

respectively, and then A6) to get

$$
11211212212122 \text { and } 111212112112122122
$$

which we can reduce using action A2).

Since all of the schedules listed above can be reduced, there are no irreducible schedules with both a 111 and a 222 . So, the schedules listed previously and their complements are the only possible schedules that cannot be reduced to better schedules, and Proposition 1 follows.

\section{B. The Optimal Schedules}

We have thus far shown that the schedules

$$
1122,11222,112222 \text {, and } 1221 \overbrace{22 \ldots 22}^{n \geq 2}
$$

are irreducible. Now we finish the proof by showing they are better than the other irreducible schedules.
Proposition 2: At any value of $p_{1}, \operatorname{EWT}\left(S, p_{1}\right)$ is minimized over all irreducible $S$ by one of

$$
1122,11222,112222 \text {, or } 1221 \overbrace{22 \ldots 22}^{n \geq 2} \text {. }
$$

To show this, we first compute their expected waiting times as a function of $p_{1}$. We then plot expected waiting time versus $p_{1}$ for each schedule. We find the appropriate intersection points and see that 1122 is best on $p_{1} \in\left(\frac{5}{16}, \frac{1}{2}\right), 11222$ is best on $\left(\frac{\tilde{5}}{21}, \frac{5}{16}\right), 112222$ is best on $\left(\frac{1}{5}, \frac{\tilde{5}}{21}\right), 122122$ is best on $\left(\frac{2}{17}, \frac{1}{5}\right)$, and

$$
122122 \overbrace{22 \ldots 22}^{n \geq 1}
$$

is best on

$$
\left(\frac{8}{(n+1)^{2}+13(n+1)+68}, \frac{8}{n^{2}+13 n+68}\right) .
$$

We compute these intersection points by first computing EWT for the schedules as a function of $p_{1}$, and $n$ when appropriate. We then set "neighboring" schedules' EWTs to equal each other and solve for $p_{1}$. We use this value of $p_{1}$ to compute the EWT and get our $\left(p_{1}\right.$, EWT) pairs.

This is illustrated in Fig. 3. The curve in the figure is the best we can do without splitting. We prove in the Appendix that the optimal schedules for two items without splitting are given by the following theorem. 
Theorem 2: For two items of lengths $l_{1}=1$ and $l_{2}=a$, the broadcast schedule that minimizes expected waiting time is

$$
\begin{aligned}
& 1^{1} 2^{1}, \text { if } p_{1} \in\left[\frac{a+1}{2 a^{2}+4 a+2}, 1-\frac{a(a+1)}{2 a^{2}+4 a+2}\right] \\
& 1^{1} 2^{n}, \quad \text { if } p_{1} \in\left[\frac{a+1}{(n+1)(n+2) a^{2}+2(n+2) a+2},\right. \\
& 1^{n} 2^{1}, \quad \text { if } p_{1} \in\left(1-\frac{a+1}{n(n+1) a^{2}+2(n+1) a+2}\right) \\
& 1-\frac{a(a+1)}{2 a^{2}+2(n+1) a+n(n+1)}, \\
&\left.1-\frac{a+1}{(n+1)(n+2) a^{2}+2(n+2) a+2}\right] .
\end{aligned}
$$

The splitting schedules 1122 and 112222 are similar to the no splitting schedules 12 and 122 , since we send the same information in the same order in each. However, the splitting schedules allow a lower EWT because we can start getting an item halfway through, unlike the no splitting schedules, where we must wait until it is sent again. As $p_{1}$ decreases, we also take advantage of the fact that we can separate the two halves of item 1 within the schedule, to get even greater gains in performance.

It remains to show that any other irreducible schedule is worse than one of the optimal schedules listed above for any value of $p_{1}$. Suppose there is some schedule, with graph defined by $t=a \cdot p_{1}+b$, that has a shorter expected waiting time at some $p_{1}$ than any of our optimal schedules. Then it will dip below the (piecewise-linear) minimum function of the graphs. Since this function is linear, it must be less at some corner of the minimum function. So, if we can show that no schedule has a graph that dips below a corner, we have shown that our schedules are optimal.

We do this by checking the set

$$
C_{1}=\left\{\left(\frac{5}{16}, \frac{7}{4}\right),\left(\frac{5}{21}, \frac{71}{42}\right),\left(\frac{1}{5}, \frac{49}{30}\right)\right\}
$$

of intersection points of $1122 \& 11222,11222 \& 112222$, and $112222 \& 122$, and the set

$C_{2}=\left\{\left(\frac{8}{n^{2}+13 n+68}, \frac{1}{2}+\frac{8\left(n^{2}+14 n+48\right)}{n^{3}+19 n^{2}+146 n+408}\right) \mid n \geq 0\right\}$

where $1221222 \overbrace{2.2}^{n}$ and $1221222 \overbrace{2.2}^{n+1}$ intersect, $\forall n \geq 0$. These are our corner points. We evaluate $t=a \cdot p_{1}+b$ at the corner point $\left(p_{c}, t_{c}\right)$ and compute the difference $t-t_{c}=a \cdot p_{c}+b-t_{c}$. We then show this is positive at all corner points.

As an example, consider the schedule $[0, N]$. If $m=N-2$, this has expected waiting time $\frac{m^{2}+7 m+10}{4(m+4)}$ for item 1 and $\frac{5}{4(m+4)}$ for item 2. So, $t-t_{c}$ at the " $n$ th" corner point in $C_{2}$ is

$$
\begin{aligned}
\frac{m^{2}+7 m}{4 m+16} \cdot \frac{8}{n^{2}+13 n+68}+ & \frac{5}{2 m+8} \\
& -\frac{4\left(n^{2}+14 n+48\right)}{n^{3}+19 n^{2}+146 n+408} .
\end{aligned}
$$

This is nonnegative $\Leftrightarrow$

$$
\begin{aligned}
& 4\left(m^{2}+7 m\right)(n+6)+5(\left.n^{3}+19 n^{2}+146 n+408\right) \\
&-4(2 m+8)\left(n^{2}+14 n+48\right) \geq 0 .
\end{aligned}
$$

We rewrite as a quadratic in $m$ to get

$$
\begin{aligned}
(4 n+24) m^{2}-\left(8 n^{2}+84 n\right. & +216) m \\
& +\left(5 n^{3}+63 n^{2}+282 n+504\right) \geq 0 .
\end{aligned}
$$

We know this is always positive if it has no real roots, so it is always positive if its discriminant is negative. So, we want

$\left(8 n^{2}+84 n+216\right)^{2}-4(4 n+24)\left(5 n^{3}+63 n^{2}+282 n+504\right)<0$.

Simplifying, we get

$$
16 n^{4}+144 n^{3}+48 n^{2}-1152 n+1728>0 .
$$

This is true for $n=0,1,2$. For $n \geq 3$, note that this expression is greater than $16(n-3)^{4}$, which is nonnegative for all $n \geq 3$. So, this expression is always positive, and hence there are no values of $m \geq 0$ and $n \geq 0$ where the schedule $[0, N]$ is better than

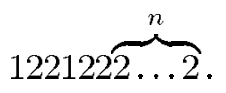

We also check against $1122,11222,112222$, and 122122 , and see that we do not dip below their corner points. We do this the same way, except now we only have the single parameter $n$ instead of both $m$ and $n$.

We use the same basic idea for all other irreducible schedules. Some of them have more than one group of $N 2$ 's. For these, we use action A5) to determine how many 2's the blocks can have relative to each other, and we consider all possible combinations, doing the above calculation for each. So, Proposition 2 is proved, and combining with Proposition 1 we see that our small set of schedules

$$
\{1122,11222,112222,1221 \overbrace{22 \ldots 22}^{n \geq 2}\}
$$

performs better than any other schedule. The intervals on which each is optimal are described by $C_{1}$ and $C_{2}$. These agree with the intervals in the statement of Theorem 1, thus completing the proof.

\section{DIFFERENT-LENGTH ITEMS}

We have shown the optimal scheduling for two items of the same length, when we split each into halves. Now we consider the same situation, but with items of different lengths. We fix the length of item 1 at $l_{1}=1$ and let item 2 have length $l_{2}$ that can be any positive value. We then split item 1 into two pieces, each of size $\frac{1}{2}$, and split item 2 into two pieces, each of size $\frac{l_{2}}{2}$. We want to find the optimal schedule for the items, as a function of the ratio of the lengths $l_{2}$ and the demand probabilities $p_{1}$ and $p_{2}=1-p_{1}$

We attempt to use the same reasoning as with $l_{2}=l_{1}$. Since the lemmas we used for equal lengths also hold true for different lengths, we can use the same reductions and arrive at the same set of irreducible schedules. However, comparing the schedules to each other is more difficult than when $l_{2}$ is fixed at 1 .

To get an idea about which schedules are optimal for which values of $l_{2}$ and $p_{1}$, we numerically checked a range of values of $l_{2}$ and $p_{1}$, and found the optimal schedule at each pair of values. The results are shown in Fig. 4. We see that the only optimal 


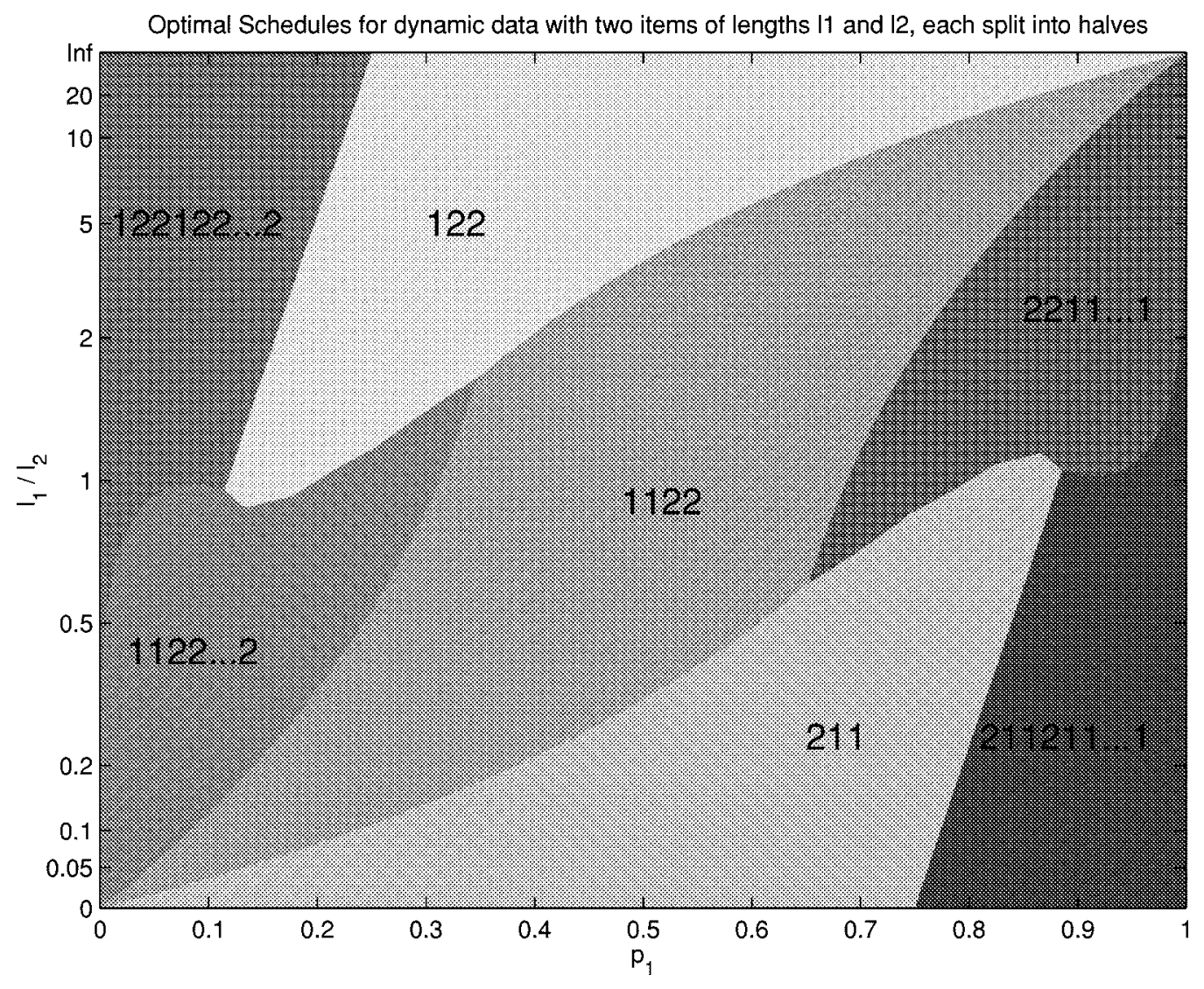

Fig. 4. Optimal scheduling with different lengths.

schedules are $1122 \ldots 2,122122 \ldots 2$, and their complements. It is interesting to note that these are the same optimal schedules as for equal-length items.

It would be interesting to know if this is provably true for all $p_{1}$ and $l_{2}$. We have been able to eliminate some of the schedules as being nonoptimal. For example, $[1, n+2]$ is always worse than $[0, n+3]$, for all $l_{2}, p_{1}$, and $n \geq 0$, so $[1, N]$ is worse than $[0, N]$ and hence $[1, N]$ is never an optimal schedule. However, we have not been able to eliminate all other schedules, as we could for $l_{2}=l_{1}$.

\section{FUTURE WORK}

Future work involves finding the best schedules for more than two items of varying lengths. We have shown that a single split can improve expected waiting time. More work can be done to see the effects of multiple splits on expected waiting time. We tried applying the ideas used in this paper for the case of splitting into three pieces, but it quickly became very complicated. It appears that different techniques or heuristics will be needed when splitting into more than two pieces. We also looked at splitting three items into halves. This also proved to be more difficult than two items split into halves. Even the case of three items without splitting is nontrivial. We would also like to look at the effects of transmission errors and ways to effectively combat them, as well as adding multiple transmission channels to improve bandwidth.

\section{APPENDIX}

PROOF OF THE LEMMAS

\section{A. Proof of Lemma 1}

1) Lemma 1 Part a):

$$
\begin{aligned}
S & =\cdots 12^{a} 12^{b} 12^{c} 1 \cdots 12^{d} 12^{e} 12^{f} 1 \cdots \\
S^{\prime} & =\cdots 12^{a} 12^{b-1} 12^{c} 1 \cdots 12^{d} 12^{e+1} 12^{f} 1 \ldots, \\
& b \geq 3, e \geq 2 .
\end{aligned}
$$

$S$ and $S^{\prime}$ have the same length $l_{S}$. We fix $l_{1}=1$ and let $l_{2}$ be any positive value. To show

$$
\operatorname{EWT}\left(S^{\prime}, p_{1}\right) \leq \operatorname{EWT}\left(S, p_{1}\right) \forall p_{1}
$$

we show

$$
\Delta t=\operatorname{EWT}\left(S, p_{1}\right)-\operatorname{EWT}\left(S^{\prime}, p_{1}\right) \geq 0 \forall p_{1} .
$$

We compute $\Delta t$ as

$$
p_{1} \Delta t_{1}+p_{2} \Delta t_{2}=p_{1}\left(t_{1}-t_{1}^{\prime}\right)+p_{2}\left(t_{2}-t_{2}^{\prime}\right)
$$

where $t_{1}$ and $t_{2}$ are the waiting times for items 1 and 2 using schedule $S$, and $t_{1}^{\prime}$ and $t_{2}^{\prime}$ are the waiting times for items 1 and 2 using schedule $S^{\prime}$. The algebra to show this follows. In each expression, we have a sum of terms of the form

$$
\text { length }\left(\text { time }_{1}+\cdots+\text { time }_{n}\right) \text {. }
$$

The length value corresponds to arriving in a section of the schedule of length length. The sum of the times is the sum of 
the lengths of the undesired sections that one waits through before getting all of the desired item, when arriving in the section of length length. Adding all terms of this form and dividing by the total length of the schedule gives us the waiting time for an item

$$
\begin{aligned}
& t_{1}=\frac{1}{l_{S}}\left[\cdots+1\left(\frac{1}{2}+a l_{2}+b l_{2}\right)+a l_{2}\left(\frac{a l_{2}}{2}+b l_{2}\right)\right. \\
& +1\left(\frac{1}{2}+b l_{2}+c l_{2}\right)+b l_{2}\left(\frac{b l_{2}}{2}+c l_{2}\right)+\cdots \\
& +1\left(\frac{1}{2}+d l_{2}+e l_{2}\right)+d l_{2}\left(\frac{d l_{2}}{2}+e l_{2}\right) \\
& \left.+1\left(\frac{1}{2}+e l_{2}+f l_{2}\right)+e l_{2}\left(\frac{e l_{2}}{2}+f l_{2}\right)+\cdots\right] \\
& t_{1}^{\prime}=\frac{1}{l_{S}}\left[\cdots+1\left(\frac{1}{2}+a l_{2}+b l_{2}-l_{2}\right)\right. \\
& +a l_{2}\left(\frac{a l_{2}}{2}+b l_{2}-l_{2}\right)+1\left(\frac{1}{2}+b l_{2}+c l_{2}-l_{2}\right) \\
& +b l_{2}\left(\frac{b l_{2}}{2}-\frac{l_{2}}{2}+c l_{2}\right)-l_{2}\left(\frac{b l_{2}}{2}-\frac{l_{2}}{2}+c_{2}\right)+\cdots \\
& +1\left(\frac{1}{2}+d l_{2}+e l_{2}+l_{2}\right)+d l_{2}\left(\frac{d l_{2}}{2}+e l_{2}+l_{2}\right) \\
& +1\left(\frac{1}{2}+e l_{2}+f l_{2}+l_{2}\right)+e l_{2}\left(\frac{e l_{2}}{2}+\frac{l_{2}}{2}+f l_{2}\right) \\
& \left.+l_{2}\left(\frac{e l_{2}}{2}+\frac{l_{2}}{2}+f l_{2}\right)+\cdots\right] \\
& \Delta t_{1}=t_{1}-t_{1}^{\prime} \\
& =\frac{1}{l_{S}}\left[1\left(l_{2}\right)+a l_{2}\left(l_{2}\right)+1\left(l_{2}\right)+b l_{2}\left(\frac{l_{2}}{2}\right)\right. \\
& +l_{2}\left(\frac{b l_{2}}{2}-\frac{l_{2}}{2}+c l_{2}\right)+1\left(-l_{2}\right)+d l_{2}\left(-l_{2}\right) \\
& \left.+1\left(-l_{2}\right)+e l_{2}\left(-\frac{l_{2}}{2}\right)-l_{2}\left(\frac{e l_{2}}{2}+\frac{l_{2}}{2}+f l_{2}\right)\right] \\
& =\frac{1}{l_{S}}\left[l_{2}^{2}(a+b+c-d-e-f-1)\right] .
\end{aligned}
$$

It is easy to see that $t_{2}=t_{2}^{\prime}$, so $\Delta t_{2}=0$. It follows that

$$
\Delta t \geq 0 \Longleftrightarrow(a+b+c)-(d+e+f) \geq 1
$$

Thus,

$$
\operatorname{EWT}\left(S^{\prime}, p_{1}\right) \leq \operatorname{EWT}\left(S, p_{1}\right) \Longleftrightarrow(a+b+c)-(d+e+f) \geq 1
$$

and part a) of the lemma is proved.

2) Lemma 1 Part b):

$$
\begin{aligned}
T & =\cdots 12^{n} 1212^{x} 12^{y} 1 \ldots \\
T^{\prime} & =\cdots 12^{n} 1122^{x} 12^{y} 1 \ldots, \quad n \geq 2, x, y \in\{0,1\} .
\end{aligned}
$$

$T$ and $T^{\prime}$ have the same length, $l_{T}$. We fix $l_{1}=1$ and let $l_{2}$ be any positive value. To show

$$
\operatorname{EWT}\left(T^{\prime}, p_{1}\right) \leq \operatorname{EWT}\left(T, p_{1}\right) \forall p_{1}
$$

we show

$$
\Delta t=\operatorname{EWT}\left(T, p_{1}\right)-\operatorname{EWT}\left(T^{\prime}, p_{1}\right) \geq 0 \forall p_{1} .
$$

We compute $\Delta t$ as

$$
p_{1} \Delta t_{1}+p_{2} \Delta t_{2}=p_{1}\left(t_{1}-t_{1}^{\prime}\right)+p_{2}\left(t_{2}-t_{2}^{\prime}\right)
$$

where $t_{1}$ and $t_{2}$ are the waiting times for items 1 and 2 using schedule $T$, and $t_{1}^{\prime}$ and $t_{2}^{\prime}$ are the waiting times for items 1 and 2 using schedule $T^{\prime}$. The algebra to show this follows. In each expression, we have a sum of terms of the form

$$
\text { length }\left(\text { time }_{1}+\cdots+\text { time }_{n}\right) \text {. }
$$

The length value corresponds to arriving in a section of the schedule of length length. The sum of the times is the sum of the lengths of the undesired sections that one waits through before getting all of the desired item, when arriving in the section of length length. Adding all terms of this form and dividing by the total length of the schedule gives us the waiting time for an item

$$
\begin{aligned}
& t_{1}=\frac{1}{l_{T}}\left[\cdots+1\left(\frac{1}{2}+n l_{2}+l_{2}\right)+n l_{2}\left(\frac{n l_{2}}{2}+l_{2}\right)\right. \\
& +1\left(\frac{1}{2}+l_{2}+x l_{2}\right)+l_{2}\left(\frac{l_{2}}{2}+x l_{2}\right) \\
& \left.+1\left(\frac{1}{2}+x l_{2}+y l_{2}\right)+\cdots\right] \\
& t_{1}^{\prime}=\frac{1}{l_{T}}\left[\cdots+1\left(\frac{1}{2}+n l_{2}\right)+n l_{2}\left(\frac{n l_{2}}{2}\right)\right. \\
& +1\left(\frac{1}{2}+l_{2}+x l_{2}\right)+1\left(\frac{1}{2}+l_{2}+x l_{2}+y l_{2}\right) \\
& \left.+l_{2}\left(\frac{l_{2}}{2}+x l_{2}+y l_{2}\right)+\cdots\right] \\
& \Delta t_{1}=t_{1}-t_{1}^{\prime} \\
& =\frac{1}{l_{T}}\left[1\left(l_{2}\right)+n l_{2}\left(l_{2}\right)+1(0)+l_{2}\left(-y l_{2}\right)+1\left(-l_{2}\right)\right] \\
& =\frac{1}{l_{T}}\left[(n-y) l_{2}^{2}\right] \\
& t_{2}=\frac{1}{l_{T}}\left[\cdots+1\left(\frac{1}{2}\right)+(n-2) l_{2}\left(\frac{l_{2}}{2}\right)+l_{2}\left(\frac{l_{2}}{2}+1\right)\right. \\
& +l_{2}\left(\frac{l_{2}}{2}+1+1+T_{2}\right)+1\left(\frac{1}{2}+1+T_{2}\right) \\
& \left.+l_{2}\left(\frac{l_{2}}{2}+1+T_{22}\right)+1\left(\frac{1}{2}+T_{22}\right)+\cdots\right] \\
& t_{2}^{\prime}=\frac{1}{l_{T}}\left[\cdots+1\left(\frac{1}{2}\right)+(n-2) l_{2}\left(\frac{l_{2}}{2}\right)+l_{2}\left(\frac{l_{2}}{2}+1+1\right)\right. \\
& +l_{2}\left(\frac{l_{2}}{2}+1+1+T_{2}\right)+1\left(\frac{1}{2}+1+T_{2}\right) \\
& \left.+1\left(\frac{1}{2}+T_{2}\right)+l_{2}\left(\frac{l_{2}}{2}+T_{22}\right)+\cdots\right]
\end{aligned}
$$




$$
\begin{aligned}
\Delta t_{2}= & t_{2}-t_{2}^{\prime} \\
= & \frac{1}{l_{T}}\left[1(0)+(n-2) l_{2}(0)+l_{2}(-1)+l_{2}(0)+1(0)\right. \\
& \left.\quad+l_{2}(1)+1\left(T_{22}-T_{2}\right)\right] \\
& \\
= & \frac{1}{l_{T}}\left[T_{22}-T_{2}\right] .
\end{aligned}
$$

Here $T_{2}$ is the time to get one piece of item 2 when we start listening at the beginning of " 2 " " and $T_{22}$ is the time to get two pieces of item 2 when we start listening at the beginning of " $2^{x}$." It is easy to see that $T_{22} \geq T_{2}$. Also, $n \geq y$, by the restrictions we placed on them. So, $\Delta t_{1} \geq 0$ and $\Delta t_{2} \geq 0$. Since $\Delta t=p_{1} \Delta t_{1}+p_{2} \Delta t_{2}$, we see that $\Delta t \geq 0$, and part b) is proved.

\section{3) Lemma 1 Part c):}

$$
\begin{aligned}
U & =\cdots 21^{r} 2^{s} 1^{t} 2^{u} 1 \ldots \\
U^{\prime} & =21^{r} 2^{s} 1^{t-1} 212^{u-1} 1 \ldots, \quad r, s \geq 1, t, u \geq 3 .
\end{aligned}
$$

$U$ and $U^{\prime}$ have the same length, $l_{U}$. We fix $l_{1}=1$ and let $l_{2}$ be any positive value. To show

$$
\operatorname{EWT}\left(U^{\prime}, p_{1}\right) \leq \operatorname{EWT}\left(U, p_{1}\right) \forall p_{1}
$$

we show

$$
\Delta t=\operatorname{EWT}\left(U, p_{1}\right)-\operatorname{EWT}\left(U^{\prime}, p_{1}\right) \geq 0 \forall p_{1} .
$$

We compute $\Delta t$ as

$$
p_{1} \Delta t_{1}+p_{2} \Delta t_{2}=p_{1}\left(t_{1}-t_{1}^{\prime}\right)+p_{2}\left(t_{2}-t_{2}^{\prime}\right)
$$

where $t_{1}$ and $t_{2}$ are the waiting times for items 1 and 2 using schedule $U$, and $t_{1}^{\prime}$ and $t_{2}^{\prime}$ are the waiting times for items 1 and 2 using schedule $U^{\prime}$. The algebra to show this follows. In each expression, we have a sum of terms of the form

$$
\text { length }\left(\text { time }_{1}+\cdots+\text { time }_{n}\right) \text {. }
$$

The length value corresponds to arriving in a section of the schedule of length length. The sum of the times is the sum of the lengths of the undesired sections that one waits through before getting all of the desired item, when arriving in the section of length length. Adding all terms of this form and dividing by the total length of the schedule gives us the waiting time for an item

$$
\begin{aligned}
t_{1}=\frac{1}{l_{U}} & {\left[\cdots+l_{2}\left(\frac{l_{2}}{2}+(r=1) s l_{2}\right)+(r>2)(r-2)\left(\frac{1}{2}\right)\right.} \\
& +(r \geq 2)\left(\frac{1}{2}+s l_{2}\right)+\left(\frac{1}{2}+s l_{2}\right) \\
& +(s>2)(s-2) l_{2}\left(\frac{(s-2) l_{2}}{2}+2 l_{2}\right) \\
& +(s \geq 2) l_{2}\left(\frac{l_{2}}{2}+l_{2}\right)+l_{2}\left(\frac{l_{2}}{2}\right)+(t-2)\left(\frac{1}{2}\right) \\
& +1\left(\frac{1}{2}+u l_{2}\right)+1\left(\frac{1}{2}+u l_{2}+T_{1}\right) \\
& \left.+u l_{2}\left(\frac{u l_{2}}{2}+T_{1}\right)+\cdots\right]
\end{aligned}
$$

$$
\begin{aligned}
t_{1}^{\prime}=\frac{1}{l_{U}} & {\left[\cdots+l_{2}\left(\frac{l_{2}}{2}+(r=1) s l_{2}\right)+(r>2)(r-2)\left(\frac{1}{2}\right)\right.} \\
& +(r \geq 2)\left(\frac{1}{2}+s l_{2}\right)+\left(\frac{1}{2}+s l_{2}\right) \\
& +(s>2)(s-2) l_{2}\left(\frac{(s-2) l_{2}}{2}+2 l_{2}\right) \\
& +(s \geq 2) l_{2}\left(\frac{l_{2}}{2}+l_{2}\right)+l_{2}\left(\frac{l_{2}}{2}\right)+(t-3)\left(\frac{1}{2}\right) \\
& +1\left(\frac{1}{2}+l_{2}\right)+1\left(\frac{1}{2}+u l_{2}\right) \\
& +l_{2}\left(\frac{l_{2}}{2}+u l_{2}-l_{2}\right)+1\left(\frac{1}{2}+u l_{2}-l_{2}+T_{1}\right) \\
& \left.+(u-1) l_{2}\left(\frac{(u-1) l_{2}}{2}+T_{1}\right)+\cdots\right]
\end{aligned}
$$$$
\Delta t_{1}=t_{1}-t_{1}^{\prime}
$$$$
=\frac{1}{l_{U}}\left[l_{2}(0)+(r>2)(r-2)(0)+(r \geq 2)(0)+(0)+(0)\right.
$$$$
+(0)+l_{2}(0)+\frac{1}{2}+(u-1) l_{2}+T_{1}+u l_{2}\left(\frac{l_{2}}{2}\right)
$$$$
+l_{2}\left(\frac{(u-1) l_{2}}{2}+T_{1}\right)-l_{2}\left(\frac{l_{2}}{2}+u l_{2}-l_{2}\right)
$$$$
\left.-1\left(\frac{1}{2}+u l_{2}-l_{2}+T_{1}\right)\right]
$$$$
=\frac{1}{l_{U}}\left[l_{2} T_{1}\right]
$$$$
t_{2}=\frac{1}{l_{U}}\left[\cdots+l_{2}\left(\frac{l_{2}}{2}+r+(s=1) t\right)+r\left(\frac{r}{2}+(s=1) t\right)\right.
$$$$
+(s>2)(s-2) l_{2}\left(\frac{l_{2}}{2}\right)+(s \geq 2) l_{2}\left(\frac{l_{2}}{2}+t\right)
$$$$
\left.+l_{2}\left(\frac{l_{2}}{2}+t\right)+t\left(\frac{t}{2}\right)+l_{2}\left(\frac{l_{2}}{2}\right)+\cdots\right]
$$$$
t_{2}^{\prime}=\frac{1}{l_{U}}\left[\cdots+l_{2}\left(\frac{l_{2}}{2}+r+(s=1)(t-1)\right)\right.
$$$$
+r\left(\frac{r}{2}+(s=1)(t-1)\right)+(s>2)(s-2) l_{2}\left(\frac{l_{2}}{2}\right)
$$$$
+(s \geq 2) l_{2}\left(\frac{l_{2}}{2}+t-1\right)+l_{2}\left(\frac{l_{2}}{2}+t\right)
$$$$
+(t-1)\left(\frac{t-1}{2}+1\right)+l_{2}\left(\frac{l_{2}}{2}+1\right)
$$$$
\left.+1\left(\frac{1}{2}\right)+\cdots\right]
$$$$
\Delta t_{2}=t_{2}-t_{2}^{\prime}
$$$$
=\frac{1}{l_{U}}\left[l_{2}((s=1) 1)+r((s=1)(1))+(s>2)(0)\right.
$$$$
\left.+(s \geq 2) l_{2}+t-2-l_{2}\right]
$$ 


$$
\begin{aligned}
& =\frac{1}{l_{U}}\left[(s \geq 2) l_{2}+(s=1)\left(l_{2}+r\right)+t-2-l_{2}\right] \\
& =\frac{1}{l_{U}}[(s=1) r+t-2] .
\end{aligned}
$$

Here $T_{1}$ is the time to get one piece of item 1 when we start listening at the end of the piece of item 1 just after " 2 " " in schedule $U$. This is the same as the time when we start listening at the end of the piece of item 1 just after " $2^{u-1}$ " in schedule $U^{\prime}$. Expressions such as $(r=1)$ and $(s>2)$ are evaluated as 1 if the expression in the parentheses is true and 0 if it is false. This is just a shorthand way of considering multiple cases with one equation. Since $T_{1} \geq 0, r \geq 1$ and $t \geq 3$, we see that $\Delta t_{1} \geq 0$ and $\Delta t_{2} \geq 0$. Since $\Delta t=p_{1} \Delta t_{1}+p_{2} \Delta t_{2}$, we see that $\Delta t \geq 0$, and part c) is proved.

4) Lemma 1 Part d):

$$
\begin{aligned}
V & =1^{a_{1}} 2^{b_{1}} 1^{a_{2}} 2^{b_{2}} \cdots 1^{a_{k-1}} 2^{b_{k-1}} 1^{a_{k}} 2^{b_{k}} \\
V^{\prime} & =2^{b_{k}} 1^{a_{k}} 2^{b_{k-1}} 1^{a_{k-1}} \cdots 2^{b_{2}} 1^{a_{2}} 2^{b_{1}} 1^{a_{1}} .
\end{aligned}
$$

Without loss of generality, we assume all $a_{i}$ 's are $1 . V$ and $V^{\prime}$ have the same length, $l_{V}$. We fix $l_{1}=1$ and let $l_{2}$ be any positive value. To show

$$
\operatorname{EWT}\left(V^{\prime}, p_{1}\right)=\operatorname{EWT}\left(V, p_{1}\right) \forall p_{1}
$$

we show

$$
\Delta t=\operatorname{EWT}\left(V, p_{1}\right)-\operatorname{EWT}\left(V^{\prime}, p_{1}\right)=0 \forall p_{1} .
$$

We compute $\Delta t$ as

$$
p_{1} \Delta t_{1}+p_{2} \Delta t_{2}=p_{1}\left(t_{1}-t_{1}^{\prime}\right)+p_{2}\left(t_{2}-t_{2}^{\prime}\right)
$$

where $t_{1}$ and $t_{2}$ are the waiting times for items 1 and 2 using schedule $V$, and $t_{1}^{\prime}$ and $t_{2}^{\prime}$ are the waiting times for items 1 and 2 using schedule $V^{\prime}$. The algebra to show this follows. In each expression, we have a sum of terms of the form

$$
\text { length }\left(\text { time }_{1}+\cdots+\text { time }_{n}\right) \text {. }
$$

The length value corresponds to arriving in a section of the schedule of length length. The sum of the times is the sum of the lengths of the undesired sections that one waits through before getting all of the desired item, when arriving in the section of length length. Adding all terms of this form and dividing by the total length of the schedule gives us the waiting time for an item

$$
\begin{aligned}
t_{1}=\frac{1}{l_{V}} \sum_{i=1}^{k}\left[1\left(\frac{1}{2}+b_{i} l_{2}+b_{(i+1) \bmod k} l_{2}\right)\right. \\
\left.+b_{i} l_{2}\left(\frac{b_{i} l_{2}}{2}+b_{(i+1) \bmod k} l_{2}\right)\right] \\
t_{1}^{\prime}=\frac{1}{l_{V}} \sum_{i=1}^{k}\left[\begin{array}{l}
b_{i} l_{2}\left(\frac{b_{i} l_{2}}{2}+b_{(i-1) \bmod k} l_{2}\right) \\
+
\end{array}\right. \\
\left.+1\left(\frac{1}{2}+b_{(i-1) \bmod k} l_{2}+b_{(i-2) \bmod k} l_{2}\right)\right]
\end{aligned}
$$

$$
\begin{aligned}
& \Delta t_{1}=t_{1}-t_{1}^{\prime} \\
& =\frac{1}{l_{V}} \sum_{i=1}^{k}\left[l_{2}\left(b_{(i+1) \bmod k}+b_{i}-b_{(i-1) \bmod k}-b_{(i-2) \bmod k}\right)\right. \\
& \left.+b_{i} l_{2}\left(b_{(i+1) \bmod k} l_{2}-b_{(i-1) \bmod k} l_{2}\right)\right] \\
& =\frac{1}{l_{V}}\left[l _ { 2 } \left(\sum_{i=1}^{k} b_{(i+1) \bmod k}+\sum_{i=1}^{k} b_{i}\right.\right. \\
& \left.-\sum_{i=1}^{k} b_{(i-1) \bmod k}-\sum_{i=1}^{k} b_{(i-2) \bmod k}\right) \\
& \left.+b_{i} l_{2}^{2}\left(\sum_{i=1}^{k} b_{(i+1) \bmod k}-\sum_{i=1}^{k} b_{(i-1) \bmod k}\right)\right] \\
& =\frac{1}{l_{V}}\left[l_{2}\left(\sum_{i=1}^{k} b_{i}+\sum_{i=1}^{k} b_{i}-\sum_{i=1}^{k} b_{i}-\sum_{i=1}^{k} b_{i}\right)\right. \\
& \left.+b_{i} l_{2}^{2}\left(\sum_{i=1}^{k} b_{i}-\sum_{i=1}^{k} b_{i}\right)\right]=0 \text {. }
\end{aligned}
$$

Similarly, $\Delta t_{2}=0$. So, we have $\Delta t=0$ and part d) is proved.

\section{B. Proof of Lemma 2}

We use the result of Lemma 1 part a), plus an additional little trick. We write $S$ as $S S S S S S$, repeating the schedule six times. This is still the same schedule, since we broadcast schedules repeatedly. Now, we choose the $b$-length section from the second $S$ and the $e$-length section from the fifth $S$, and choose $a$ and $c$ adjacent to $b$, and $d$ and $f$ adjacent to $e$ in the overall schedule. There can be no overlap of these regions. We now apply Lemma 1 part a) to $S S S S S S$ to get $S S^{-} S S S^{+} S$. Here $S^{-}$is $S$ with $b$ decreased by one and $S^{+}$is $S$ with $e$ increased by one. We will write $S^{ \pm}$to represent $S$ with $b$ decreased by one and $e$ increased by one. We then do a cyclic shift and repeat on $S^{-} S S S^{+} S S$ to get $S^{-} S^{-} S S^{+} S^{+} S$. We shift and repeat four more times, giving

$$
S^{ \pm} S^{ \pm} S^{ \pm} S^{ \pm} S^{ \pm} S^{ \pm}=S^{ \pm}=S^{\prime}
$$

in Lemma 1 part a). At each step, we reduced EWT, so

$$
\operatorname{EWT}\left(S^{\prime}, p_{1}\right) \leq \operatorname{EWT}\left(S, p_{1}\right) \forall p_{1}
$$

and Lemma 2 is proved.

\section{Proof of Lemma 3}

Since $S$ repeats cyclically, we can assume without loss of generality that it starts with one of the subschedules $C$. We can write schedule $S$ as

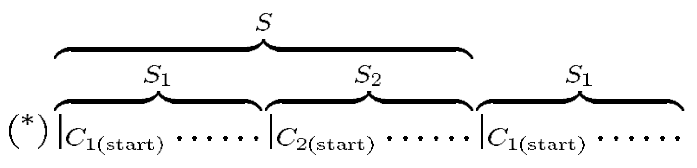

where $C_{1 \text { (start) }}$ indicates the start of the first instance of subschedule $C$ within $S$ and $C_{2 \text { (start) }}$ indicates the start of the second occurrence. 
We can also write schedules $S_{1}$ and $S_{2}$ as follows:

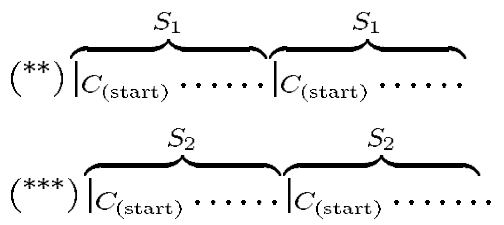

If we start waiting at some time within the first $S_{1}$ group in either $\left(^{*}\right)$ or $\left({ }^{* *}\right)$, we will wait through a certain number of blocks before finding enough blocks of the desired item. If we stay within the initial $S_{1}$ group, the times are identical for $\left(^{*}\right)$ and $\left({ }^{* *}\right)$ since the sequence of blocks that we encounter is the same in each case. If we must proceed into the next group, we first enter the $C$ subschedule in either case. Since $C$ contains two blocks each of items 1 and 2, we will not have to advance past the $C$ group, so the waiting times in these cases are identical, since we again encounter the same sequence of blocks. So, it follows that the waiting time for schedule $S$, given that we arrive somewhere within subschedule $S_{1}$, is the same as it is for $S_{1}$ treated as a full schedule. The same applies for subschedule $S_{2}$. So, we have

$\operatorname{EWT}\left(S, p_{1}\right)=\operatorname{Pr}\left(\right.$ arrive in $\left.S_{1}\right) \cdot \operatorname{EWT}\left(S_{1}, p_{1}\right)$
$+\operatorname{Pr}\left(\right.$ arrive in $\left.S_{2}\right) \cdot \operatorname{EWT}\left(S_{2}, p_{1}\right)$.

The probabilities of arriving in subschedules $S_{1}$ and $S_{2}$ within schedule $S$ are simply $\frac{l_{S_{1}}}{l_{S}}$ and $\frac{l_{S_{2}}}{l_{S}}$, respectively. So, we have

$$
\operatorname{EWT}\left(S, p_{1}\right)=\frac{l_{S_{1}}}{l_{S}} \cdot \operatorname{EWT}\left(S_{1}, p_{1}\right)+\frac{l_{S_{2}}}{l_{S}} \cdot \operatorname{EWT}\left(S_{2}, p_{1}\right) .
$$

\section{Proof of Theorem 2}

1) Lemma for Theorem 2: First, we prove a result for no splitting similar to Lemma 1 part c) for splitting.

Lemma 4: Let schedule $S=1^{m+2} 2^{n+2}$ and schedule $S^{\prime}=$ $1^{m+1} 212^{n+1}$. Then

$$
\operatorname{EWT}\left(S^{\prime}, p_{1}\right)<\operatorname{EWT}\left(S, p_{1}\right) \forall m, n \geq 0, a>0 .
$$

We have two schedules $S=1^{m+2} 2^{n+2}$ and $S^{\prime}=$ $1^{m+1} 212^{n+1}$. We compute the expected waiting times for each item for each schedule, and compare these. We then compare the overall expected waiting times for the schedules

$$
\begin{aligned}
t_{1}= & m\left(\frac{1}{2}\right)+1\left(\frac{1}{2}\right)+1\left(\frac{1}{2}+2 a+n a\right) \\
& +a\left(\frac{a}{2}+a+n a\right)+a\left(\frac{a}{2}+n a\right)+n a\left(\frac{n a}{2}\right) \\
t_{1}^{\prime}= & m\left(\frac{1}{2}\right)+1\left(\frac{1}{2}+a\right) a\left(\frac{a}{2}\right) \\
& +1\left(\frac{1}{2}+a+n a\right)+a\left(\frac{a}{2}+n a\right)+n a\left(\frac{n a}{2}\right) \\
\Delta t_{1}= & t_{1}-t_{1}^{\prime} \\
= & m(0)+1(-a)+1(a)+a(a+n a)+a(0)+n a(0) \\
= & a^{2}+n a^{2}>0 \quad \forall m, n, a
\end{aligned}
$$

$$
\begin{aligned}
t_{2}= & m\left(\frac{m}{2}+2\right)+1\left(\frac{1}{2}+1\right)+1\left(\frac{1}{2}\right) \\
& +n a\left(\frac{a}{2}\right)+a\left(\frac{a}{2}\right)+a\left(\frac{a}{2}+m+2\right) \\
t_{2}^{\prime}= & m\left(\frac{m}{2}+1\right)+1\left(\frac{1}{2}\right)+a\left(\frac{a}{2}+1\right) \\
& +1\left(\frac{1}{2}\right)+n a\left(\frac{a}{2}\right)+a\left(\frac{a}{2}+m+1\right) \\
\Delta t_{2}= & t_{2}-t_{2}^{\prime} \\
= & m(1)+1(1)+1(0)+a(-1)+n a(0)+a(1) \\
= & m+1>0 \quad \forall m, n, a \\
\Delta t= & \Delta t_{1} p_{1}+\Delta t_{2} p_{2}>0 \quad \forall m, n, a .
\end{aligned}
$$

So, it follows that

$$
\text { EWT }\left(S^{\prime}, p_{1}\right)<\operatorname{EWT}\left(S, p_{1}\right) \forall m, n \geq 0, a>0 .
$$

2) Proof of Theorem 2: First we note that it is never optimal to have a schedule with two occurrences of 12 (or 21). This follows from Lemma 3. So the optimal schedule is always a sequence of 1's followed by a sequence of 2's. But if we have the sequence 1122 , it is better to replace it with 1212 , according to the lemma above. So, the optimal schedule is one of $122 \ldots 2$ or $11 \ldots 12$. The expected waiting time for $122 \ldots 2$ is

$$
t_{n}=(n-1) \frac{n a^{2}+2 a}{2(n a+1)} p_{1}+\frac{n a^{2}+2 a+1}{2(n a+1)} .
$$

We see that $t_{n}=t_{n+1}$ at

$$
p_{1}^{n, n+1}=\frac{a+1}{n(n+1) a^{2}+2(n+1) a+2} .
$$

The expected waiting time for $11 \ldots 12$ is

$$
t_{n}=(n-1) \frac{n a^{2}+2 a}{2(n a+1)}\left(1-p_{1}\right)+\frac{n a^{2}+2 a+1}{2(n a+1)} .
$$

This is just symmetric to the other schedules.

We check the waiting time of each of these schedules at the values of $p_{1}$ above and find that they are all greater than or equal to the corresponding times of the intersecting schedules at these points. So, the best schedules are the ones we indicated on the indicated ranges of $p_{1}$.

\section{REFERENCES}

[1] D. Aksoy and M. J. Franklin, "Scheduling for large-scale on-demand data broadcasting," in Proc. IEEE INFOCOM, San Francisco, CA, Mar. 1998.

[2] A. Bar-Noy, R. Bhatia, J. Naor, and B. Schieber, "Minimizing service and operations cost of periodic scheduling," in Proc. 9th Annu. ACM-SIAM Symp. Discrete Algorithms (SODA), 1998.

[3] A. Bestavros, "AIDA-based real-time fault-tolerant broadcast disks," Dept. Comput. Sci., Boston Univ., Boston, MA, Tech. Rep. 96-001, Jan. $5,1996$.

[4] M. J. Franklin and S. Zdonik, "Dissemination-based information systems," IEEE Data Eng. Bull., vol. 19, no. 3, Sept. 1996.

[5] S. Hameed, "Scheduling information broadcast in asymmetric environment," M. Sci. thesis, Texas A\&M Univ., College Station, May 1997. 
[6] S. Hameed and N. H. Vaidya, "Log-time algorithms for scheduling single and multiple channel data broadcast," in Proc. MOBICOM'97, Budapest, Hungary, Sept. 1997.

[7] S. Jiang and N. H. Vaidya, "Scheduling algorithms for a data broadcast system: Minimizing variance of the response time," Dept. Comput. Sci., Texas A\&M Univ., College Station, Tech. Rep. 98-005, Feb. 4, 1998.

[8] H. V. Leong and A. Si, "Database caching over the air-storage," Comput. J., vol. 40, no. 7, pp. 401-415, 1997.

[9] N. H. Vaidya and S. Hameed, "Data broadcast in asymmetric wireless environments," in Proc. 1st Int. Workshop Satellite-Based Information Services (WOSBIS), Rye, NY, Nov. 1996.
[10] L. Xu, "Efficient and scalable on-demand data streaming using UEP codes," presented at the ACM Multimedia 2001 Conf., Ottowa, ON, Canada, Oct. 2001.

[11] S. Zdonik, M. J. Franklin, R. Alonso, and S. Acharya, "Are 'Disks in the Air' just 'Pie in the Sky'?," in Proc. IEEE Workshop Mobile Computing Systems and Applications, Santa Cruz, CA, Dec. 1994. 